\title{
Dissociable Representations of Environmental Size and Complexity in the Human Hippocampus
}

\author{
Oliver Baumann ${ }^{1}$ and Jason B. Mattingley ${ }^{1,2}$ \\ ${ }^{1}$ Queensland Brain Institute, and ${ }^{2}$ School of Psychology, The University of Queensland, St. Lucia 4072, Queensland, Australia
}

The hippocampus is widely assumed to play a central role in representing spatial layouts in the form of "cognitive maps." It remains unclear, however, which properties of the world are explicitly encoded in the hippocampus, and how these properties might contribute to the formation of cognitive maps. Here we investigated how physical size and complexity, two key properties of any environment, affect memory-related neural activity in the human hippocampus. We used functional magnetic resonance imaging and a virtual maze-learning task to examine retrieval-related activity for three previously learned virtual mazes that differed systematically in their physical size and complexity (here defined as the number of distinct paths within the maze). Before scanning, participants learned to navigate each of the three mazes; hippocampal activity was then measured during brief presentations of static images from within each maze. Activity within the posterior hippocampus scaled with maze size but not complexity, whereas activity in the anterior hippocampus scaled with maze complexity but not size. This double dissociation demonstrates that environmental size and complexity are explicitly represented in the human hippocampus, and reveals a functional specialization for these properties along its anterior-posterior axis.

\section{Introduction}

Humans, like many animals, possess a remarkable ability to navigate their way to a desired but currently unobservable location using an internal representation of the external world. Such internal representations of large-scale spaces are typically referred to as cognitive maps (Tolman, 1948), and the hippocampus is thought to play a pivotal role in their construction and maintenance (O'Keefe and Nadel, 1978; Burgess et al., 2002). Although it is widely agreed that the hippocampus is able to construct and maintain internal representations of space (but see Eichenbaum et al., 1999; Shrager et al., 2008), it is not known precisely which properties of the external world are explicitly encoded and ultimately form part of the cognitive map. Changes in environmental size and geometry can cause alterations in the firing of hippocampal and entorhinal neurons (Muller and Kubie, 1987; O'Keefe and Burgess, 1996; Sharp, 1999). More specifically, the presence and properties of extended surfaces or boundaries exert a powerful influence on activity patterns in the wider hippocampal formation in humans and rodents (O'Keefe and Burgess, 1996; Yoganarasimha and Knierim, 2005; Savelli et al., 2008; Solstad et al., 2008; Lever et al., 2009; Bird et al., 2010), suggesting that

\footnotetext{
Received Jan. 24, 2013; revised May 12, 2013; accepted May 17, 2013.

Author contributions: $0 . B$. and J.B.M. designed research; 0. B. performed research; $0 . B$. analyzed data; $0 . B$ and J.B.M. wrote the paper.

This work was supported by an Australian Research Council (ARC) Thinking Systems Grant. O.B. was supported by an ARC Discovery Early Career Researcher Award (DE120100535) and a UQ Foundation Research Excellence Award. J.B.M. was supported by an ARC Australian Laureate Fellowship (FL110100103).

Correspondence should be addressed to Oliver Baumann, Queensland Brain Institute, The University of Queensland, St. Lucia 4072, QLD, Australia. E-mail: 0.baumann@uq.edu.au.

DOI:10.1523/JNEUROSCI.0350-13.2013

Copyright $\odot 2013$ the authors $\quad 0270-6474 / 13 / 3310526-08 \$ 15.00 / 0$
}

structural properties of environments are key components of mental representations of space.

Here we investigated whether two key properties of the physical environment-its size and complexity (defined here as the number of distinct paths contained within it) — are represented as unique patterns of activation within the human hippocampus. Further, since previous investigations of the role of the hippocampus in spatial cognition and memory have suggested a degree of functional specialization along its anterior-posterior axis (Colombo et al., 1998; Kühn and Gallinat, 2013; Nadel et al., 2013), we aimed to quantify retrieval-related activity separately for its anterior (head) and posterior (tail) subregions. In rodents, it has been observed that the size of place fields increases from posterior to anterior hippocampus (Jung et al., 1994; Kjelstrup et al., 2008). Computationally, large place fields are ideally suited to contribute to path-planning operations (Hok et al., 2005), whereas the execution of these plans is likely to benefit from access to high-resolution representations provided by small place fields. Based on these findings, we hypothesized that increases in environmental size would lead to proportionally larger or more energy intensive point-by-point representations of space in the posterior hippocampus, whereas a higher number of navigational choice points should pose a higher load on path-planning operations mediated by the anterior hippocampus.

Our study consisted of two phases: an initial learning phase, in which participants actively navigated three different virtual mazes that differed systematically in their size and complexity; and a subsequent retrieval phase, undertaken in the scanner, during which participants determined to which maze a single, static image belonged. We compared the retrieval-related functional magnetic resonance imaging (fMRI) responses for 
a

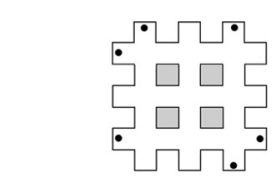

C
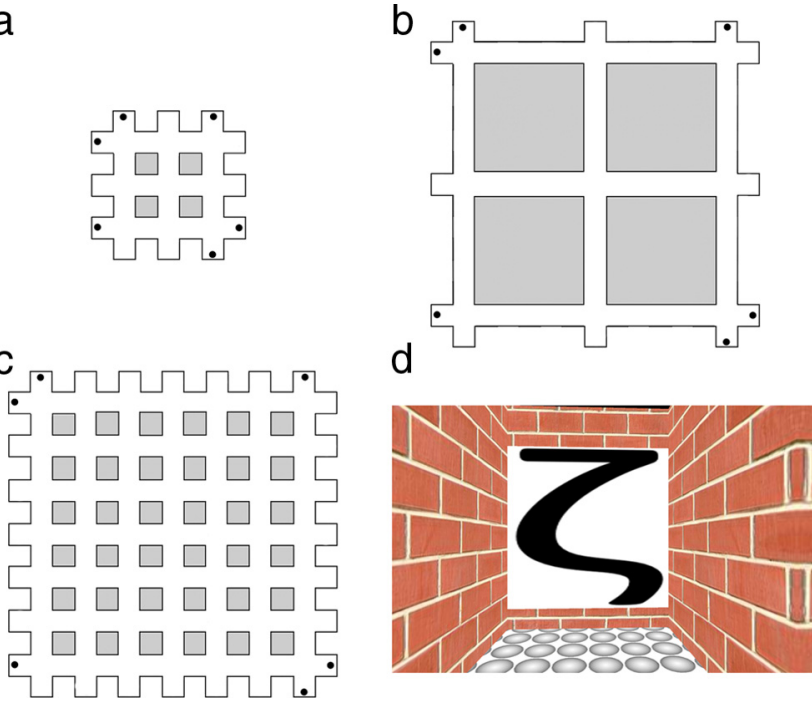

Figure 1. Schematics of the virtual environment and spatial classification task. $\boldsymbol{a}-\boldsymbol{c}$, Aerial perspective of the three virtual mazes used in the learning phase. Critically, participants only viewed the mazes from a first-person perspective. The black dots indicate the locations of the symbols that acted as landmarks. $\boldsymbol{d}$, Example of a single image of a landmark viewed by participants during the retrieval phase.

the different mazes to determine whether environmental size and complexity are detectable as unique and dissociable patterns of activation within the anterior and posterior subdivisions of the human hippocampus.

\section{Materials and Methods}

\section{Participants}

Eighteen healthy, adult volunteers gave their informed consent to participate in the study, which was approved by the Human Research Ethics Committee of The University of Queensland. The participants (nine females) ranged in age from 18 to 28 years (mean age, 22 years); all were right handed.

\section{Behavioral training and fMRI study}

To test for neural responses associated with variations in environmental size and complexity, we developed a navigation task in which participants first familiarized themselves with the layout of the three virtual mazes (the learning phase). In the MRI scanner they then viewed images of landmarks from these mazes and were asked to retrieve which of the three mazes the landmark belonged to (the retrieval phase).

Learning phase. Participants completed two $1 \mathrm{~h}$ training sessions, which consisted of a series of search tasks that required them to navigate as efficiently as possible to various landmarks positioned at the periphery of the virtual mazes (Fig. $1 a-c$ ). We used the Blender open-source threedimensional content creation suite (The Blender Foundation) to create the virtual maze and to administer the learning task. All three mazes were Cartesian grids, but they varied in the number and length of the corridors they contained. Mazes A and B consisted of 6 corridors, whereas Maze C consisted of 14 corridors. Further, the corridors in Maze A had a (virtual) length of $28 \mathrm{~m}$, whereas the corridors in Mazes B and C had a length of $64 \mathrm{~m}$. Therefore, Mazes A and B differed in the minimum distance between target locations (i.e., size), whereas Mazes B and C differed in the number of possible routes between target locations (i.e., complexity). The ends of selected corridors contained a landmark in the form of a distinct black symbol on a white background. The symbols were letters from the Tagalog and Greek alphabets, as well as astronomical signs, which were randomized across the three mazes and participants, to avoid any confounding effects that might arise as a consequence of particular groupings. The maze environment was constructed so that participants were never able to see more than one landmark at a time as they moved along the corridors. This ensured that the relationship between the positions of the landmarks had to be encoded entirely on the basis of an internal, allocentric representation of the layout of the virtual maze. Critically, the number of discrete landmarks per maze $(N=6)$ and their categorical arrangement (always located in the outside corners of the grid) were held constant. We also informed participants about this arrangement, to minimize the likelihood of individual differences in search behavior in the initial training blocks for the three mazes.

The participants' task was to navigate as efficiently as possible to the location of one of the six landmarks in each maze, indicated by a small cue (an image of the landmark) at the top of the computer screen. In every trial, participants started from a different position, directly facing one of the landmarks. At the end of each trial, the efficiency of the path taken by the participant was measured in terms of the distance traversed. This information was flashed to participants after each trial to motivate them to improve their performance and thus to become more familiar with the spatial layout of the virtual maze. In each of the two training sessions, participants completed two blocks of 30 trials for each maze in an alternating fashion (e.g., $\mathrm{ABCABC}$ ). In every block of trials, participants had to navigate to each of the six landmarks five times in random order. The order in which the three mazes were learned was counterbalanced across participants, as were the labels that were attributed to them (i.e., Maze A, Maze B, and Maze C).

Retrieval phase. In the retrieval phase, participants viewed static, snapshot images of the 18 landmarks they had seen previously across the three different mazes during the learning phase, while undergoing fMRI. As shown in Figure $1 d$, the static views from the maze depicted only the landmark and did not show any aspects of the maze geometry (i.e., its size or its number of corridors), which might otherwise have revealed its identity. They were instructed to press one of three buttons using their right index finger to indicate which of the three mazes the landmark belonged to (i.e., A, B, or C). Each image was presented for $2 \mathrm{~s}$ followed by a blank screen for $4-8 \mathrm{~s}$. To avoid any neural or behavioral adaptation or repetition effects (Krekelberg et al., 2006), participants were never shown consecutive landmarks from the same maze. Before the fMRI experiment, participants completed 20 training trials to familiarize themselves with the task. In the fMRI experiment, participants completed four scanning runs, for a total of 14 trials for each of the landmarks and a total of 84 trials for each of the mazes. The sequencing of the trials, and the temporal jittering of the intertrial blank periods, was optimized with optseq2 software (http://surfer.nmr.mgh.harvard.edu/optseq/).

MRI acquisition and data analysis. Brain images were acquired on a $3 \mathrm{~T}$ MR scanner (Trio; Siemens) fitted with a 32-channel head coil. For the functional data, 33 axial slices (slice thickness, $2 \mathrm{~mm}$; interslice gap, 1 $\mathrm{mm}$ ) were acquired in a descending order, using a gradient echo echoplanar $2^{*}$-sensitive sequence (repetition time, $2.06 \mathrm{~s}$; echo time, $30 \mathrm{~ms}$; flip angle, $90^{\circ}$; matrix, $64 \times 64$; field of view, $192 \times 192 \mathrm{~mm}$; in-plane resolution, $3 \times 3 \mathrm{~mm}$; phase encoding polarity, positive). In each session, 337 volumes were acquired for each participant; the first four images were discarded to allow for T1 equilibration. The sequence was optimized to minimize signal dropouts in the medial temporal lobes (Weiskopf et al., 2006), and geometric distortions in the EPI images caused by magnetic field inhomogeneities were corrected using a point-spread mapping approach (Zeng and Constable, 2002; Zaitsev et al., 2003). We also acquired a T1-weighted structural MPRAGE scan. A liquid crystal display projector back-projected images from the virtual environment onto a screen positioned at the head end of the scanner gantry. Participants lay on their back within the bore of the magnet and viewed the stimuli via a mirror that reflected the images displayed on the screen. To minimize head movement, all participants were stabilized with tightly packed foam padding surrounding the head.

Image processing and statistical analyses were performed using SPM8 (Wellcome Department of Imaging Neuroscience, University College London, London, UK). Functional data volumes were slice-time corrected and realigned to the first volume. A T2*-weighted mean image of the unsmoothed images was coregistered with the corresponding anatomical T1-weighted image from the same individual. The individual T1 image was used to derive the transformation parameters for the stereo- 


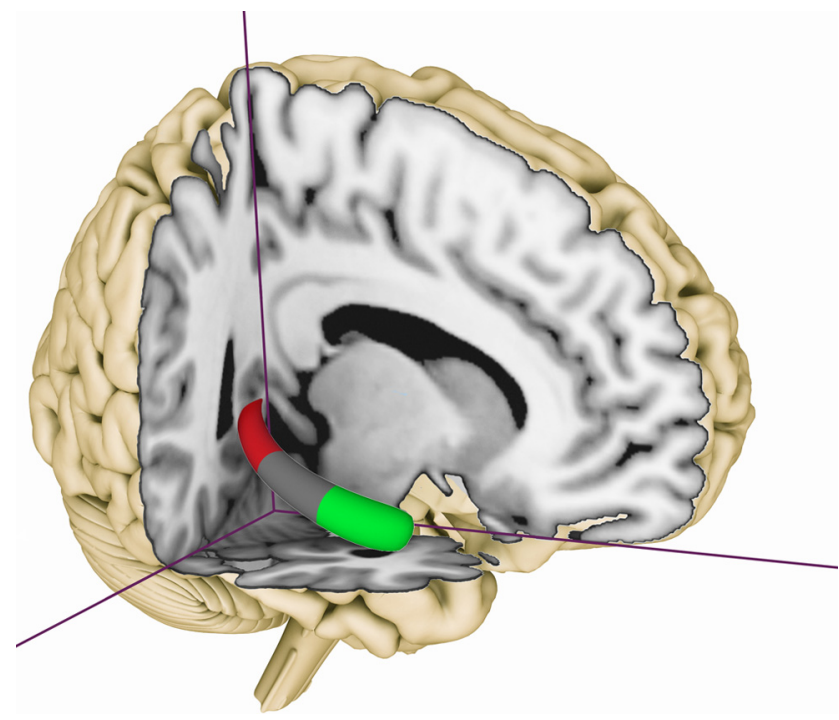

Figure 2. Anatomical locations of the two regions of interest [right anterior hippocampus (head), green; right posterior hippocampus (tail), red].

taxic space using the SPM8 template [Montreal Neurological Institute (MNI) template], which was then applied to the individual coregistered EPI images. We aimed to increase the sensitivity of our fMRI analyses using a hypothesis-driven and targeted region of interest (ROI) analysis. First, we restricted our analysis to the right hippocampus, given that previous literature has frequently identified a right-hemispheric preponderance for tasks involving spatial memory and navigation (Maguire et al., 1997, 1998; Bohbot et al., 1998; Spiers et al., 2001; Iaria et al., 2003; Fortin et al., 2008; Gagnon et al., 2012; Nedelska et al., 2012; Kühn and Gallinat, 2013). Second, recent human and rodent studies have suggested a segregation of the hippocampus into three anatomically and functionally distinct subregions: an anterior region (the head), an intermediate region (body), and a posterior region (tail) (Fanselow and Dong, 2010; Brown et al., 2012).

Given that previous research has focused on the anterior and posterior segments of the hippocampus, we created two ROIs that encompassed the hippocampal head and tail sections, but not the intermediate body section (Fig. 2). To ensure generalizability, we began by using the hippocampal template from the Anatomical Automatic Labeling (AAL) atlas ROI library (Tzourio-Mazoyer et al., 2002). For each participant, we then divided the hippocampus into three sections, as follows. We determined the anterior border of the hippocampal tail $(y=-30 \pm 0.7)$ and the posterior border of the hippocampal head $(y=-16 \pm 1.4)$ in the participants' normalized structural images using the approaches advocated by Pruessner et al. (2000) and Malykhin et al. (2007). This approach strikes an optimal balance between generalizability (achieved through use of the AAL atlas) and specificity (by considering individual differences in hippocampal morphology). We calculated the percentage of signal change as an index of effect size for retrieval-related activity for each of the three mazes, separately for all individual participants, for the anterior and posterior hippocampal ROIs, using the MarsBaR toolbox (http://marsbar.sourceforge.net/).

To avoid cross-contamination between the two ROIs and the adjacent hippocampal body and other nearby structures, we used normalized but unsmoothed fMRI data. We restricted our ROI analyses to these two regions - head and tail - to reduce the overall number of statistical comparisons, and because the relevant literature permits clear-cut predictions for these two anatomical subregions. The visual stimulation periods were modeled as $2 \mathrm{~s}$ events, separately for the three different maze conditions. The percentage signal change for the different ROIs was subjected to repeated-measures $2 \times 2$ ANOVAs, with post hoc $t$ tests for related samples, using a stringent statistical threshold ( $p=0.05$, corrected for multiple comparisons). For completeness, and to assess any potential contribution from the hippocampal body and other

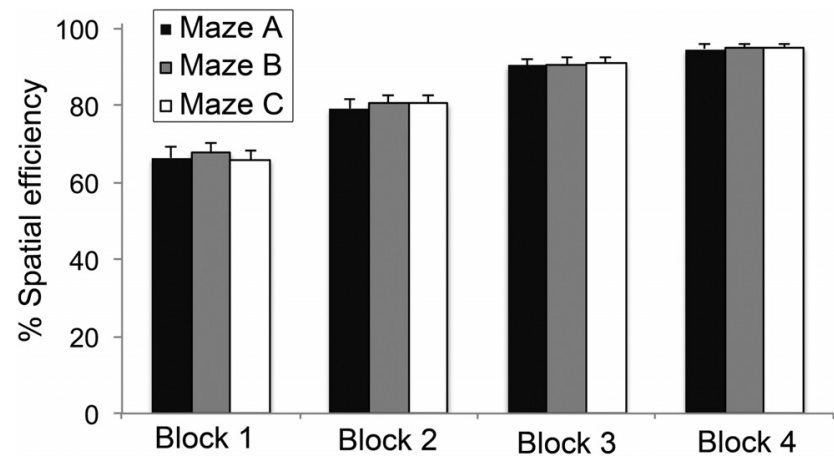

Figure 3. Mean values for spatial efficiency during the learning phase, expressed as a percentage of the optimal path length ( $\pm 1 \mathrm{SE}$ ) for the three mazes across the four learning blocks.

cortical regions, we also report the results from an exploratory wholebrain analysis on the effects of manipulating maze size and complexity. For this analysis, we smoothed the images with an $8 \mathrm{~mm}$ fullwidth half maximum isotropic Gaussian kernel and used a liberal threshold of $p=0.005$ (uncorrected for multiple comparisons; 15 continuous voxels).

\section{Results}

\section{Behavioral data}

As participants learned the layout of the virtual mazes across the two training sessions (six training blocks per session), we recorded the spatial efficiency of the paths they took to reach the cued landmark. Spatial efficiency was defined as the ratio of the minimum distance between the starting point and the target landmark, and the actual length of the path taken by the participant. Throughout training, the participants gradually improved their ability to reach the target locations in an efficient manner (Fig. 3). We also observed that, as training progressed, participants increasingly relied on a small set of routes to travel between the goal locations. In the final training block, participants obtained an average spatial efficiency of $94.72 \%(\mathrm{SE}=1.03 \%)$ in Maze A, of $95.17 \%(\mathrm{SE}=1.05 \%)$ in Maze B, and of $95.11 \%(\mathrm{SE}=$ $0.97 \%$ ) in Maze C. To further assess navigational strategies for the three mazes, we determined the proportion of trials in which participants traversed the inner versus the outer corridors of the mazes. For all three mazes, $\sim 99 \%$ of paths traversed by participants involved the outer corridors only. Thus, although participants had several alternative routes available to them to reach each goal location, they tended to use only a limited subset of them. This pattern was not entirely unexpected, because we informed participants that target stimuli would be always located in the corners of the maze to minimize strategic differences. We return to consider the potential relevance of this behavioral pattern and its neural correlates in the Discussion. Together, these results indicate that participants learned to navigate all three mazes equally well, independent of their physical size and complexity.

Statistical analyses of the behavioral data acquired during the retrieval phase showed that there was no difference in the average time required by participants to identify the landmarks from the three different mazes (Maze $\mathrm{A}=1.01 \mathrm{~s}, \mathrm{SE}=0.10 \mathrm{~s}$; Maze $\mathrm{B}=1.04 \mathrm{~s}, \mathrm{SE}=0.09 \mathrm{~s}$; Maze $\mathrm{C}=1.01 \mathrm{~s}, \mathrm{SE}=0.10 \mathrm{~s}$; repeated-measures ANOVA, two-tailed $F_{(2,17)}=0.99, p=$ $0.382)$. Moreover, there was no difference in performance accuracy across the three mazes (Maze $\mathrm{A}=89.74 \%, \mathrm{SE}=2.98 \%$; Maze $\mathrm{B}=88.49 \%, \mathrm{SE}=3.20 \%$; Maze $\mathrm{C}=91.04 \%$, $\mathrm{SE}=$ $2.86 \%$; repeated-measures ANOVA, two-tailed $F_{(2,17)}=0.70$, 


\section{a Effects of size}

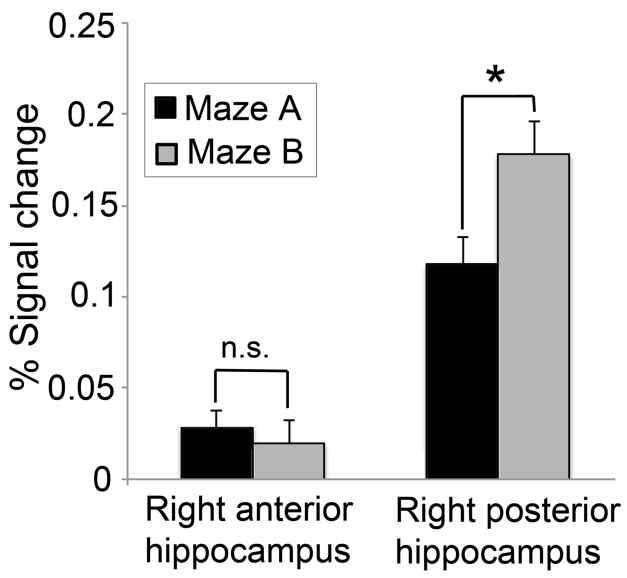

\section{b Effects of complexity}

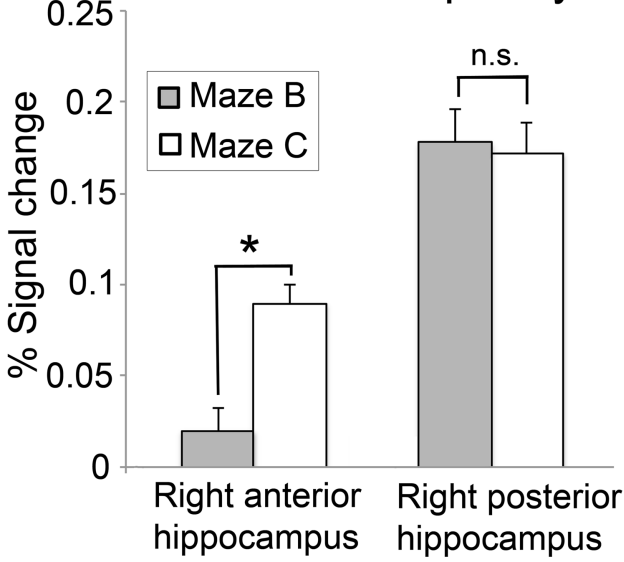

Figure 4. Regionally averaged percentage BOLD signal change ( $\pm 1 \mathrm{SE})$ for the right anterior and right posterior hippocampal regions of interest during the retrieval phase; ${ }^{*}$ significant, $p \leq$ 0.05 (paired $t$ tests, corrected for multiple comparisons). $\boldsymbol{a}$, Effects of environmental size (Maze B $>$ Maze A). $\boldsymbol{b}$, Effects of environmental complexity (Maze C > Maze B).

$p=0.504)$. Together, these findings show that, behaviorally, maze size and complexity had no significant effect on participants' performance in the retrieval phase.

\section{fMRI data}

The main focus of our analyses was on activity within the anterior head section and posterior tail section of the right hippocampus (Fig. 2), which are homologues of the ventral and dorsal hippocampal formation in rats. Our principal comparisons for the fMRI analysis involved the factors of maze size and maze complexity. We used the MarsBaR toolbox (http://marsbar. sourceforge.net/) to calculate the percentage signal change as an index of effect size for retrieval-related blood oxygenation level-dependent (BOLD) activity for each of the three mazes, separately for all participants, for each of the two right hippocampal ROIs (see Fig. 4). Extracted percentage signal change for the ROIs was subjected to two separate, repeatedmeasures $2 \times 2$ ANOVAs to determine the relative contributions of environmental size and complexity on hippocampal activity.

The first ANOVA, with factors of size (small or large) and region (right anterior and posterior hippocampus), revealed a significant main effect for region $\left(F_{(1,17)}=137.478 ; p<0.001\right)$, as well as a marginally significant main effect of size $\left(F_{(1,17)}=4.005\right.$; $p=0.062)$. Critically, there was also a significant interaction between region and size $\left(F_{(1,17)}=9.254 ; p=0.007\right)$. Post hoc, paired $t$ tests revealed that while activity in the hippocampal tail was positively related to maze size $\left(t_{(17)}=2.895 ; p=0.010\right)$, this was not the case for the hippocampal head $\left(t_{(17)}=-0.657 ; p=\right.$ 0.520. A second ANOVA, with factors of complexity (low or high) and region (right anterior and posterior hippocampus), revealed significant main effects for region $\left(F_{(1,17)}=123.587 ; p<\right.$ $0.001)$ and complexity $\left(F_{(1,17)}=4.766 ; p=0.043\right)$, as well as a significant interaction between them $\left(F_{(1,17)}=6.134 ; p=0.024\right)$. Post hoc paired $t$ tests revealed that while activity in the hippocampal head was positively related to maze complexity $\left(t_{(17)}=\right.$ 6.689; $p<0.001)$, this was not the case for the hippocampal tail $\left(t_{(17)}=-0.208 ; p=0.838\right)$. Together, therefore, the results reveal a classic double dissociation, such that activity within the right posterior hippocampus is related to maze size but not complexity, whereas activity in the right anterior hippocampus is related to maze complexity but not size. The main effect of region observed in both ANOVAs was due to a generally higher signal in the hippocampal tail compared with the hippocampal head, mostly likely caused, at least in part, by susceptibility-induced signal loss in the anterior hippocampus (Greicius et al., 2003).

Finally, to test for activations associated with the manipulation of maze size and complexity in other brain areas involved in encoding and retrieval of spatial environments, including the parietal, parahippocampal and retrosplenial cortex, as well as the striatum (Rosenbaum et al., 2004; Janzen and van Turennout, 2004; Wolbers and Büchel, 2005; Bohbot et al., 2007; Iaria et al., 2007; Doeller et al., 2008; Baumann et al., 2010, 2012; Schinazi and Epstein, 2010), we conducted an exploratory whole-brain analysis using a more liberal threshold of $p=0.005$ (uncorrected; 15 continuous voxels). For the effect of size (Maze B > Maze A), this analysis revealed four regions of gray matter activation (see Fig. 5), which were located in the left ectosplenial and retrosplenial cortices $(-6,-46,20$; 95 voxels; Brodmann areas 26, 29, and 30; see Vogt et al., 2001, for anatomical labels) and right ectosplenial cortex $(12,-46,26 ; 41$ voxels; Brodmann area 26), the left hippocampus $(-18,-36,6 ; 75$ voxels), and the right hippocampus $(32,-30,-4 ; 37$ voxels). The left hippocampal activation cluster was limited to the tail section, whereas the right hippocampal cluster encompassed tail as well as body sections. For the effect of complexity (Maze $\mathrm{C}>$ Maze B), the analysis revealed an activity cluster in the right anterior hippocampus, which extended into the middle parahippocampal gyrus (28, $-14,-22$; 181 voxels; Fig. 6).

Overall, then, the exploratory whole-brain analysis confirmed the pattern observed in the ROI analysis, implicating the posterior hippocampus in the representation of maze size, and the anterior hippocampus in representing maze complexity. In addition, the whole-brain analysis suggests that both the right and left posterior hippocampus are modulated by environmental size. Finally, we found that activity in the retrosplenial cortex, a region that has been implicated in the representation of spatial layouts (Harker and Whishaw, 2004; Wolbers and Büchel, 2005; Iaria et al., 2007), was related to maze size, but not complexity.

\section{Discussion}

We have shown that activity in the human hippocampus is modulated by the size and complexity (number of paths) of a virtual environment. When participants viewed images from the two larger mazes, activity in the posterior hippocampus was significantly elevated bilaterally, but there was no activation change in 


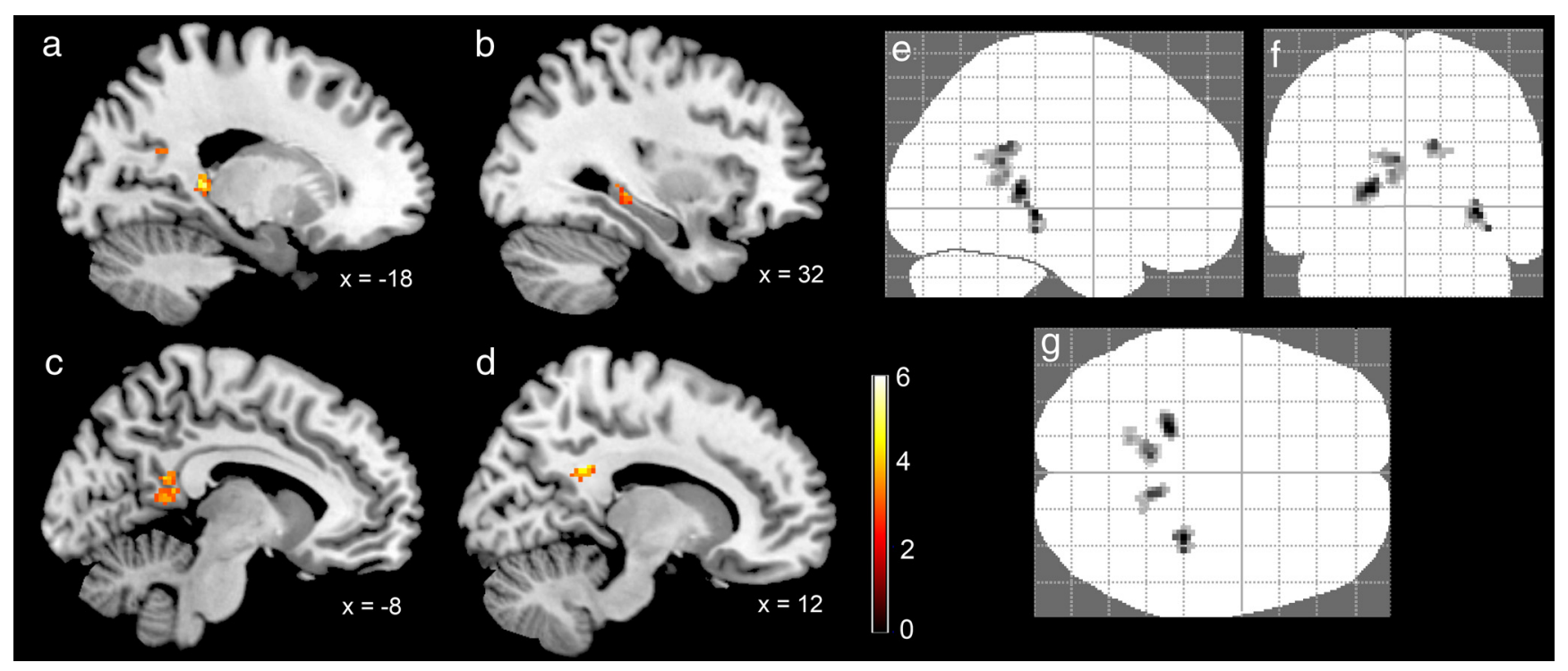

Figure 5. Mean BOLD activity from the exploratory whole-brain analysis ( $p=0.005$; uncorrected; 15 continuous voxels) of the effects of maze size (Maze B $>$ Maze A). Sagittal MR slices from an MNI-normalized template brain show significant activity in a number of brain regions. $\boldsymbol{a}$, Left posterior hippocampus. $\boldsymbol{b}$, Right posterior and intermediate hippocampus. $\boldsymbol{c}$, Left ectosplenial and retrosplenial cortex. $\boldsymbol{d}$, Right ectosplenial cortex. $\boldsymbol{e}-\boldsymbol{g}$, "Glass brains" showing the location and size of all activation clusters.

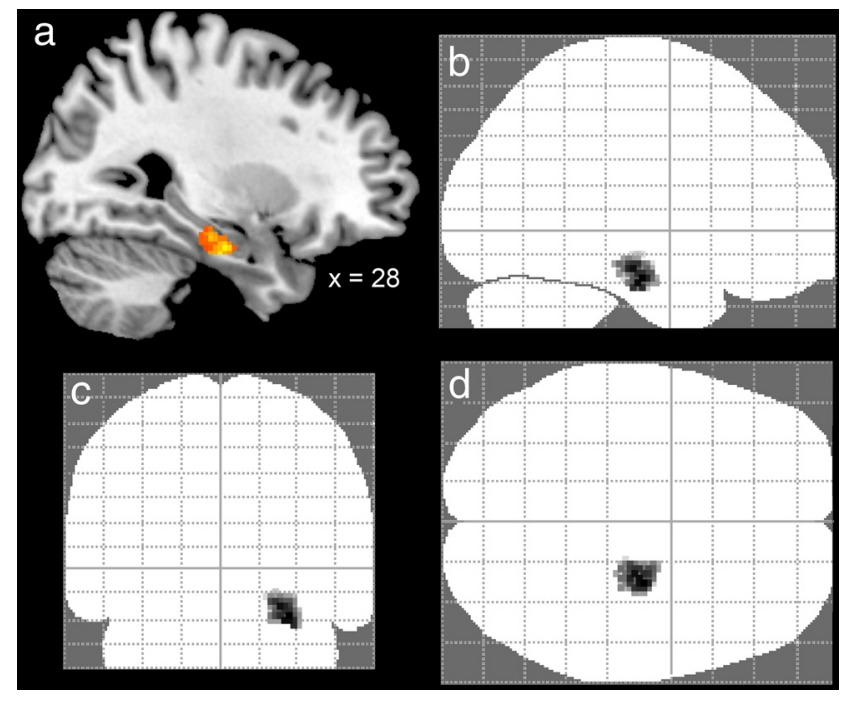

Figure 6. Mean BOLD activity from the exploratory whole-brain analysis ( $p=0.005$; uncorrected; 15 continuous voxels) of the effects of maze complexity (Maze ( $>$ Maze B). $\boldsymbol{a}$, Sagittal brain slice showing significant activity in the right anterior hippocampus. $\boldsymbol{b}$ - $\boldsymbol{d}$, "Glass brains" showing the location and size of the hippocampal activation cluster.

the anterior hippocampus. By contrast, when participants viewed images from the maze that contained a greater number of navigational choice points, neural activity was elevated in the right anterior hippocampus, but there was no such effect in the posterior hippocampus. We observed these effects even though during retrieval participants were shown only static images of landmarks from the mazes, none of which contained any aspect of the maze geometry (i.e., its size or the number of corridors). This suggests that the landmarks acted as partial input cues driving the hippocampus to complete the associated spatial representation of the entire maze, consistent with previous literature on hippocampal functioning (Mizumori et al., 1989; O’Reilly et al., 1998).

Most importantly, our finding of enhanced right hippocampal activation during retrieval of the larger and more complex mazes also suggests that these important environmental proper- ties are represented by proportionally larger or more energyintensive neural networks. Notably, statistical analyses of the behavioral data acquired during the retrieval phase revealed no effect of complexity or size on retrieval speed or accuracy, suggesting that these two parameters did not affect the degree of memory strength for, or familiarity with, the landmarks during the retrieval phase. Together, our findings provide compelling support for the idea that the right hippocampus is specifically involved in constructing and maintaining spatial representations of the environment (O'Keefe and Nadel, 1978; Maguire et al., 1999; Burgess et al., 2002).

Although several human neuroimaging investigations and patient studies (Maguire et al., 1998; Ekstrom et al., 2003; Spiers and Maguire, 2006; Bohbot et al., 2007; Iaria et al., 2007; Wolbers et al., 2007; Hassabis et al., 2009; Brown et al., 2012) have identified the hippocampus as being pivotal in the formation and use of internal representations of real-world and virtual space, it still remains largely unknown which properties of environments are explicitly encoded in the hippocampus, and ultimately form part of the human cognitive map. Recent human imaging studies have shown that extended surfaces or boundaries, in contrast to single discrete landmarks, exert a powerful influence on hippocampal activity and hippocampally mediated spatial learning (Doeller et al., 2008; Bird et al., 2010), suggesting that structural and geometric properties of environments are key components of our mental representations of space (Stankiewicz and Kalia, 2007). The present study extends these earlier suggestions by revealing that the key environmental properties of size and complexity are strong modulators of spatial memory-related hippocampal activity patterns, suggesting that they are explicitly encoded in human internal representations of the world.

The present findings also illustrate an important functional dissociation within the hippocampus for the representation of different environmental properties along its anterior-posterior axis. Specifically, we found that the spatial scale of the environment was indexed bilaterally in the posterior (tail) section of the hippocampus, whereas the number of navigational choice points was indexed in the right anterior (head) section. Several studies have suggested a functional specialization along the anterior- 
posterior axis of the hippocampus. Most commonly, it had been proposed that the posterior hippocampus is involved in spatial learning and navigation (for an overview, see Kühn and Gallinat (2013)), whereas the anterior hippocampus is thought to be involved in a broader range of functions, including involvement in episodic memory (for an overview, see Kühn and Gallinat (2013)) and novelty detection (Strange et al., 1999). However, whereas those previous studies mainly explored hippocampal dissociations across cognitive domains (but see Brown et al., 2012), our study investigated the contributions of anterior and posterior subregions solely within the domain of spatial navigation. Our data suggest a model of hippocampal functioning in which its anterior and posterior sections subserve different roles in maintaining representations of spatial environments and, ultimately, for successful navigation. That neural activity in the posterior hippocampus was positively related with the spatial scale of the environment suggests that it might store a metrically proportional model of the external environment, which corresponds approximately point by point (i.e., like an analog representation) to the physical environment. However, since the presence of additional corridors in Maze $\mathrm{C}$ did not affect the posterior hippocampal signal, this representation is likely to include only those corridors that were actually traversed by participants (i.e., that were task relevant). Hence, retrieval-related activity in the posterior hippocampus appears to scale with the lengths of the paths traversed during navigation in the learning phase, rather than with the actual physical size of the maze. As mentioned in the Results, even though especially Maze $\mathrm{C}$ offered several alternative routes to navigate between goal locations, as training progressed, participants would typically use only a subset of them. In contrast, we found that the number of navigational choice points (but not its spatial scale) reliably modulated retrieval-related activity in the anterior hippocampus. Therefore, in contrast to the posterior hippocampus, the anterior segment of the hippocampus might simply store an index, or a global schematic representation, of all potential routes and navigational choice points between goal locations.

Interestingly, in rodents, it had been observed that place field size increases from dorsal to ventral (Jung et al., 1994; Kjelstrup et al., 2008). Computationally, large place fields would be ideally suited to path-planning operations (Hok et al., 2005), whereas the execution of these plans is likely to benefit from access to high-resolution representations provided by small place fields. Similarly, two more recent navigation studies in humans have suggested that while the posterior hippocampus is particularly involved in the retrieval of detailed and fine-grained spatial information, the anterior hippocampus appears to be involved in the retrieval of more global or contextual representations of the spatial environment (Hirsshorn et al., 2012; Nadel et al., 2013). Even though such observations are open to multiple functional interpretations, they are nevertheless consistent with our taskspecific model of hippocampal function, in which its anterior section stores a global but schematic representation of all available routes or choice points in an environment, and its posterior section provides a detailed analog representation of task-relevant sections of the environment.

Our model of spatial representations within the hippocampus also dovetails with several earlier findings from human imaging studies. The posterior hippocampus is typically active whenever spatial associations have to be formed in a way that allows for absolute metric accuracy during navigation (Hartley et al., 2003; Baumann et al., 2010, 2012), or when previously learned information is reinstated in its original form
(Giovanello et al., 2009; Xu et al., 2010). In contrast, the anterior hippocampus is active during goal-directed spatial planning, representing goal proximity and indexing of path accuracy (Maguire et al., 2003; Morgan et al., 2011; Viard et al., 2011). All these studies are in line with a model of hippocampal function in which the posterior section maintains stable and detailed spatial representations, and the anterior section stores more integrated but coarse representations, which support the flexible planning of routes. Nevertheless, further experiments are necessary to explicitly test this proposed model. For example, it will be important to determine whether the presence of navigational barriers, which forces participants to use alternative routes between goal locations and therefore increases the task-relevant sections of the maze, leads to an increase in the size of the posterior hippocampal representation.

In conclusion, we have shown that neural signatures of two key properties of the external environment-size and complexity - can be measured in activity patterns from the human hippocampus. Our findings support the idea that, as in rodents, the human hippocampus encodes and maintains models of spatial environments. The data further highlight a major functional dissociation between anterior and posterior hippocampal representations of spatial layouts. Our findings therefore support the idea of information-rich but compartmentalized hippocampal representations of space. It remains to be seen how other environmental properties, such as the number of task-relevant and task-irrelevant object landmarks, are encoded in the human hippocampus. It will also be important to investigate to what degree human cognitive maps rely upon, and work in concert with, extrahippocampal structures, including the parahippocampal and retrosplenial cortices, whose activity patterns are not only modulated by the geometry of spatial environments (Janzen and van Turennout, 2004; Epstein, 2008), but also by navigational performance and expertise (Wolbers and Büchel, 2005; Baumann et al., 2010).

\section{References}

Baumann O, Chan E, Mattingley JB (2010) Dissociable neural circuits for encoding and retrieval of object locations during active navigation in humans. Neuroimage 49:2816-2825. CrossRef Medline

Baumann O, Chan E, Mattingley JB (2012) Distinct neural networks underlie encoding of categorical versus coordinate spatial relations during active navigation. Neuroimage 60:1630-1637. CrossRef Medline

Bird CM, Capponi C, King JA, Doeller CF, Burgess N (2010) Establishing the boundaries: the hippocampal contribution to imagining scenes. J Neurosci 30:11688-11695. CrossRef Medline

Bohbot VD, Kalina M, Stepankova K, Spackova N, Petrides M, Nadel L (1998) Spatial memory deficits in patients with lesions to the right hippocampus and to the right parahippocampal cortex. Neuropsychologia 36:1217-1238. CrossRef Medline

Bohbot VD, Lerch J, Thorndycraft B, Iaria G, Zijdenbos AP (2007) Gray matter differences correlate with spontaneous strategies in a human virtual navigation. J Neurosci 27:10078-10083. CrossRef Medline

Brown TI, Ross RS, Tobyne SM, Stern CE (2012) Cooperative interactions between hippocampal and striatal systems support flexible navigation. Neuroimage 60:1316-1330. CrossRef Medline

Burgess N, Maguire EA, O'Keefe J (2002) The human hippocampus and spatial and episodic memory. Neuron 35:625-641. CrossRef Medline

Colombo M, Fernandez T, Nakamura K, Gross CG (1998) Functional differentiation along the anterior-posterior axis of the hippocampus in monkeys. J Neurophysiol 80:1002-1005. Medline

Doeller CF, King JA, Burgess N (2008) Parallel striatal and hippocampal systems for landmarks and boundaries in spatial memory. Proc Natl Acad Sci U S A 105:5915-5920. CrossRef Medline

Eichenbaum H, Dudchenko P, Wood E, Shapiro M, Tanila H (1999) The 
hippocampus, memory, and place cells: is it spatial memory or a memory space? Neuron 23:209-226. CrossRef Medline

Ekstrom AD, Kahana MJ, Caplan JB, Fields TA, Isham EA, Newman EL, Fried I (2003) Cellular networks underlying human spatial navigation. Nature 425:184-188. CrossRef Medline

Epstein RA (2008) Parahippocampal and retrosplenial contributions to human spatial navigation. Trends Cogn Sci 12:388-396. CrossRef Medline

Fanselow MS, Dong HW (2010) Are the dorsal and ventral hippocampus functionally distinct structures? Neuron 65:7-19. CrossRef Medline

Fortin M, Voss P, Lord C, Lassonde M, Pruessner J, Saint-Amour D, Rainville C, Lepore F (2008) Wayfinding in the blind: larger hippocampal volume and supranormal spatial navigation. Brain 131:2995-3005. CrossRef Medline

Gagnon L, Schneider FC, Siebner HR, Paulson OB, Kupers R, Ptito M (2012) Activation of the hippocampal complex during tactile maze solving in congenitally blind subjects. Neuropsychologia 50:1663-1671. CrossRef Medline

Giovanello KS, Schnyer D, Verfaellie M (2009) Distinct hippocampal regions make unique contributions to relational memory. Hippocampus 19:111-117. CrossRef Medline

Greicius MD, Krasnow B, Boyett-Anderson JM, Eliez S, Schatzberg AF, Reiss AL, Menon V (2003) Regional analysis of hippocampal activation during memory encoding and retrieval: fMRI study. Hippocampus 13:164-174. CrossRef Medline

Harker KT, Whishaw IQ (2004) Impaired place navigation in place and matching-to-place swimming pool tasks follows both retrosplenial cortex lesions and cingulum bundle lesions in rats. Hippocampus 14:224-231. CrossRef Medline

Hartley T, Maguire EA, Spiers HJ, Burgess N (2003) The well-worn route and the path less traveled: distinct neural bases of route following and wayfinding in humans. Neuron 37:877-888. CrossRef Medline

Hassabis D, Chu C, Rees G, Weiskopf N, Molyneux PD, Maguire EA (2009) Decoding neuronal ensembles in the human hippocampus. Curr Biol 19:546-554. CrossRef Medline

Hirshhorn M, Grady C, Rosenbaum RS, Winocur G, Moscovitch M (2012) Brain regions involved in the retrieval of spatial and episodic details associated with a familiar environment: an fMRI study. Neuropsychologia 50:3094-3106. CrossRef Medline

Hok V, Save E, Lenck-Santini PP, Poucet B (2005) Coding for spatial goals in the prelimbic/infralimbic area of the rat frontal cortex. Proc Natl Acad Sci U S A 102:4602-4607. CrossRef Medline

Iaria G, Petrides M, Dagher A, Pike B, Bohbot VD (2003) Cognitive strategies dependent on the hippocampus and caudate nucleus in human navigation: variability and change with practice. J Neurosci 23:5945-5952. Medline

Iaria G, Chen JK, Guariglia C, Ptito A, Petrides M (2007) Retrosplenial and hippocampal brain regions in human navigation: complementary functional contributions to the formation and use of cognitive maps. Eur J Neurosci 25:890-899. CrossRef Medline

Janzen G, van Turennout M (2004) Selective neural representation of objects relevant for navigation. Nat Neurosci 7:673-677. CrossRef Medline

Jung MW, Wiener SI, McNaughton BL (1994) Comparison of spatial firing characteristics of units in dorsal and ventral hippocampus of the rat. J Neurosci 14:7347-7356. Medline

Kjelstrup KB, Solstad T, Brun VH, Hafting T, Leutgeb S, Witter MP, Moser EI, Moser MB (2008) Finite scale of spatial representation in the hippocampus. Science 321:140-143. CrossRef Medline

Krekelberg B, Boynton GM, van Wezel RJ (2006) Adaptation: from single cells to BOLD signals. Trends Neurosci 29:250-256. CrossRef Medline

Kühn S, Gallinat J (2013) Segregating cognitive functions within hippocampal formation: a quantitative meta-analysis on spatial navigation and episodic memory. Hum Brain Mapp. Advance online publication. Retrieved May 25, 2013. doi:10.1002/hbm.22239. CrossRef Medline

Lever C, Burton S, Jeewajee A, O'Keefe J, Burgess N (2009) Boundary vector cells in the subiculum of the hippocampal formation. J Neurosci 29:97719777. CrossRef Medline

Maguire EA, Frackowiak RS, Frith CD (1997) Recalling routes around London: activation of the right hippocampus in taxi drivers. J Neurosci 17: 7103-7110. Medline

Maguire EA, Burgess N, Donnett JG, Frackowiak RS, Frith CD, O'Keefe J (1998) Knowing where and getting there: a human navigation network. Science 280:921-924. CrossRef Medline
Maguire EA, Burgess N, O’Keefe J (1999) Human spatial navigation: cognitive maps, sexual dimorphism, and neural substrates. Curr Opin Neurobiol 9:171-177. CrossRef Medline

Maguire EA, Spiers HJ, Good CD, Hartley T, Frackowiak RS, Burgess N (2003) Navigation expertise and the human hippocampus: a structural brain imaging analysis. Hippocampus 13:250-259. CrossRef Medline

Malykhin NV, Bouchard TP, Ogilvie CJ, Coupland NJ, Seres P, Camicioli R (2007) Three-dimensional volumetric analysis and reconstruction of amygdala and hippocampal head, body and tail. Psychiatry Res 155:155165. CrossRef Medline

Mizumori SJ, McNaughton BL, Barnes CA, Fox KB (1989) Preserved spatial coding in hippocampal CA1 pyramidal cells during reversible suppression of CA3c output: evidence for pattern completion in hippocampus. J Neurosci 9:3915-3928. Medline

Morgan LK, Macevoy SP, Aguirre GK, Epstein RA (2011) Distances between real-world locations are represented in the human hippocampus. J Neurosci 31:1238-1245. CrossRef Medline

Muller RU, Kubie JL (1987) The effects of changes in the environment on the spatial firing of hippocampal complex-spike cells. J Neurosci 7:19511968. Medline

Nadel L, Hoscheidt S, Ryan LR (2013) Spatial cognition and the hippocampus: the anterio-posterio axis. J Cogn Neurosci 25:22-28. CrossRef Medline

Nedelska Z, Andel R, Laczó J, Vlcek K, Horinek D, Lisy J, Sheardova K, Bures J, Hort J (2012) Spatial navigation impairment is proportional to right hippocampal volume. Proc Natl Acad Sci U S A 109:2590-2594. CrossRef Medline

O'Keefe J, Burgess N (1996) Geometric determinants of the place fields of hippocampal neurons. Nature 381:425-428. CrossRef Medline

O'Keefe J. Nadel L (1978) The hippocampus as a cognitive map. Oxford, UK: Clarendon.

O'Reilly RC, Norman KA, McClelland JL (1998) A hippocampal model of recognition memory. In: Advances in neural information processing systems 10 (Jordan MI, Kearns MJ, Solla SA, eds), pp 73-79. Cambridge: MIT.

Pruessner JC, Li LM, Serles W, Pruessner M, Collins DL, Kabani N, Lupien S, Evans AC (2000) Volumetry of hippocampus and amygdala with highresolution MRI and three-dimensional analysis software: minimizing the discrepancies between laboratories. Cereb Cortex 10:433-442. CrossRef Medline

Rosenbaum RS, Ziegler M, Winocur G, Grady CL, Moscovitch M (2004) "I have often walked down this street before": fMRI studies on the hippocampus and other structures during mental navigation of an old environment. Hippocampus 14:826-835. CrossRef Medline

Savelli F, Yoganarasimha D, Knierim JJ (2008) Influence of boundary removal on the spatial representations of the medial entorhinal cortex. Hippocampus 18:1270-1282. CrossRef Medline

Schinazi VR, Epstein RA (2010) Neural correlates of real-world route learning. Neuroimage 53:725-735. CrossRef Medline

Sharp PE (1999) Subicular place cells expand or contract their spatial firing pattern to fit the size of the environment in an open field but not in the presence of barriers: comparison with hippocampal place cells. Behav Neurosci 113:643-662. CrossRef Medline

Shrager Y, Kirwan CB, Squire LR (2008) Neural basis of the cognitive map: path integration does not require hippocampus or entorhinal cortex. Proc Natl Acad Sci U S A 105:12034-12038. CrossRef Medline

Solstad T, Boccara CN, Kropff E, Moser MB, Moser EI (2008) Representation of geometric borders in the entorhinal cortex. Science 322:18651868. CrossRef Medline

Spiers HJ, Maguire EA (2006) Thoughts, behaviour, and brain dynamics during navigation in the real world. Neuroimage 31:1826-1840. CrossRef Medline

Spiers HJ, Burgess N, Maguire EA, Baxendale SA, Hartley T, Thompson PJ, O'Keefe J (2001) Unilateral temporal lobectomy patients show lateralized topographical and episodic memory deficits in a virtual town. Brain 124:2476-2489. CrossRef Medline

Stankiewicz BJ, Kalia AA (2007) Acquisition of structural versus object landmark knowledge. J Exp Psychol Hum Percept Perform 33:378-390. CrossRef Medline

Strange BA, Fletcher PC, Henson RN, Friston KJ, Dolan RJ (1999) Segregating the functions of human hippocampus. Proc Natl Acad Sci U S A 96: 4034-4039. CrossRef Medline 
Tolman EC (1948) Cognitive maps in rats and men. Psychol Rev 55:189-208. CrossRef Medline

Tzourio-Mazoyer N, Landeau B, Papathanassiou D, Crivello F, Etard O, Delcroix N, Mazoyer B, Joliot M (2002) Automated anatomical labeling of activations in SPM using a macroscopic anatomical parcellation of the MNI MRI single-subject brain. Neuroimage 15:273-289. CrossRef Medline

Viard A, Doeller CF, Hartley T, Bird CM, Burgess N (2011) Anterior hippocampus and goal-directed spatial decision making. J Neurosci 31:46134621. CrossRef Medline

Vogt BA, Vogt LJ, Perl DP, Hof PR (2001) Cytology of human caudomedial cingulate, retrosplenial, and caudal parahippocampal cortices. J Comp Neurol 438:353-376. CrossRef Medline

Weiskopf N, Hutton C, Josephs O, Deichmann R (2006) Optimal EPI parameters for reduction of susceptibility-induced BOLD sensitivity losses: a wholebrain analysis at $3 \mathrm{~T}$ and $1.5 \mathrm{~T}$. Neuroimage 33:493-504. CrossRef Medline

Wolbers T, Büchel C (2005) Dissociable retrosplenial and hippocampal contributions to successful formation of survey representations. J Neurosci 25:3333-3340. CrossRef Medline

Wolbers T, Wiener JM, Mallot HA, Büchel C (2007) Differential recruitment of the hippocampus, medial prefrontal cortex, and the human motion complex during path integration in humans. J Neurosci 27: 9408-9416. CrossRef Medline

Xu J, Evensmoen HR, Lehn H, Pintzka CW, Håberg AK (2010) Persistent posterior and transient anterior medial temporal lobe activity during navigation. Neuroimage 52:1654-1666. CrossRef Medline

Yoganarasimha D, Knierim JJ (2005) Coupling between place cells and head direction cells during relative translations and rotations of distal landmarks. Exp Brain Res 160:344-359. CrossRef Medline

Zaitsev M, Steinhoff S, Shah NJ (2003) Error reduction and parameter optimization of the TAPIR method for fast T1 mapping. Magn Reson Med 49:1121-1132. CrossRef Medline

Zeng H, Constable RT (2002) Image distortion correction in EPI: comparison of field mapping with point spread function mapping. Magn Reson Med 48:137-146. CrossRef Medline 